\title{
Erratum to: Development of SCAR markers for rapid and specific detection of Pseudomonas syringae pv. morsprunorum races 1 and 2, using conventional and real-time PCR
}

\author{
Monika Kałużna $^{1}$ • Pedro Albuquerque ${ }^{2,3}$ - Fernando Tavares $^{2,3} \cdot$ Piotr Sobiczewski $^{1}$. \\ Joanna Puławska ${ }^{1}$
}

Published online: 20 December 2016

(C) Springer-Verlag Berlin Heidelberg 2016

Erratum to: This complements the above paper in Appl Microbiol Biotechnol, volume 100 on page 3705

DOI 10.1007/s00253-016-7295-0

In the original version of this article inadvertently there is a lack of the information concerning the annealing temperature used for primers designed for conventional PCR.

It should be written:

For the primers designed for conventional PCR, among the tested annealing temperatures, $59^{\circ} \mathrm{C}$ for primers Psm1-6F and Psm1-6R (for detection of $P s m 1$ strains) and $54^{\circ} \mathrm{C}$ for primers Psm2-8F and Psm2-8R (for detection of Psm2 strains) were selected as the optimal ones.

The online version of the original article can be found at doi:10.1007/s00253016-7295-0.

Monika Kałużna

monika.kaluzna@inhort.pl

Research Institute of Horticulture, Konstytucji 3 Maja 1/3,

96-100 Skierniewice, Poland

2 Centro de Investigação em Biodiversidade e Recursos Genéticos (CIBIO), InBIO, Laboratório Associado, Universidade do Porto, Campus Agrário de Vairão, 4485-661 Vairão, Portugal

3 Faculdade de Ciencias, Departamento de Biologia, Universidade do Porto, Edifício FC4, Via Panoramica No. 36,

4150-564 Porto, Portugal 